\title{
BMJ Open Role of locoregional surgery in treating FIGO 2009 stage IVB cervical cancer patients: a population-based study
}

\author{
Yanhong Wang (D) , ${ }^{1}$ Yi Ouyang, ${ }^{2}$ Jingjing Su, ${ }^{1}$ Zhigang Bai, ${ }^{1}$ Qunrong Cai, ${ }^{1}$ \\ Xinping $\mathrm{Cao}^{2}$
}

To cite: Wang Y, Ouyang $Y$, Su J, et al. Role of locoregional surgery in treating FIGO 2009 stage IVB cervical cancer patients: a populationbased study. BMJ Open 2021;11:e042364. doi:10.1136/ bmjopen-2020-042364

- Prepublication history and additional supplemental material for this paper are available online. To view these files, please visit the journal online. (http://dx.doi.org/10.1136/ bmjopen-2020-042364).

$\mathrm{YW}$ and $\mathrm{YO}$ contributed equally.

Received 08 July 2020 Accepted 21 July 2021

\section{Check for updates}

(C) Author(s) (or their employer(s)) 2021. Re-use permitted under CC BY-NC. No commercial re-use. See rights and permissions. Published by BMJ.

${ }^{1}$ Radiotherapy, Second Affiliated Hospital of Fujian Medical University, Quanzhou, Fujian, China

${ }^{2}$ Radiotherapy Department, State Key Laboratory of Oncology in South China; Sun Yat-sen University Cancer Center; State Key Laboratory of Oncology in South China; Collaborative Innovation Center for Cancer Medicine, Guangzhou, Guangdong, China

Correspondence to

Qunrong Cai;

249211337@qq.com

\section{ABSTRACT}

Objective We aimed to analyse the clinical value of primary site surgery in improving the cancer-specific survival (CSS) and overall survival (OS) of initial metastatic cervical cancer patients.

Design A population-based retrospective study.

Setting National Cancer Institute's Surveillance, Epidemiology and End Results database.

Participants We analysed 1390 patients with the International Federation of Gynecology and Obstetrics 2009 stage IVB cervical cancer with complete clinical data treated between 2010 and 2016.

Interventions Primary site surgery.

Measures Propensity score matching (PSM) with a ratio of 1:2 was used to balance measure covariates of comparison groups. Survival time was calculated using Kaplan-Meier methods and compared by the log-rank test. To eliminate the bias of site-specific metastasis, clinicopathological factors and subsequent therapy on survival analysis, subgroup analyses stratified by metastasis type, clinicopathological factors and subsequent therapy were employed to evaluate the effect of cervical surgery on survival. Combination of directed acyclic graph and change-in-estimate procedures was performed to indentified confounders, and Cox regression was used to assess the survival benefit of cervical surgery for primary metastatic cervical cancer patients. The consistency of our findings was evaluated through sensitivity analysis.

Results Matching resulted in two comparison groups with minor differences in most variables. Pre-and-post-PSM, the median CSS and OS in the surgery group were 1.3 and $1.5,1.1$ and 1.2 times of those in the non-surgery group, respectively. Primary site surgery conferred prognosis superiority for patients with metastases to distant lymph node and other sites rather than organ metastases. After PSM and adjusting confounders, local surgery reduced the cancer related and overall mortality rates by $31 \%$ and $30 \%$, respectively.

Conclusions Surgical procedures could promote survival in patients with primary metastatic cervical cancer and should be considered a therapeutic option for carefully chosen patients.

\section{INTRODUCTION}

Cervical cancer remains one of the most common tumours affecting women

\section{Strengths and limitations of this study}

An inherent selection bias existed due to the nature of this population-based retrospective study.

- Propensity score matching (PSM) was done to balance the measured covariates between the two groups.

- Combination of directed acyclic graph and changein-estimate was used to identify the confounders, and multivariate Cox regression analysis was performed to adjust confounders.

- Sensitivity analysis was performed based on data of another PSM with matching ratio 1:3 to evaluate the results consistency.

- The details of chemotherapy, radiation and recovery time following surgery were not available in the Surveillance, Epidemiology and End Result database, which could also affect prognosis.

worldwide, ranking third in cancer incidence and fourth in mortality. ${ }^{1}$ Among patients with newly diagnosed cervical cancer, roughly $10 \%$ have distant metastasis at their initial diagnosis and are staged as International Federation of Gynecology and Obstetrics (FIGO) 2009 stage IVB, ${ }^{2}$ and have a dismal prognosis, with poor median survival time usually less than $1-2$ years. ${ }^{34}$

For primary metastatic cervical cancer, platinum-based chemotherapy, the angiogenesis inhibitor bevacizumab and immunotherapy are the choice of treatment and considered palliative. Moreover, studies on locoregional radiation therapy combined with system therapy for primary metastatic cervical cancer are emerging and have shown that locoregional radiotherapy would confer a substantially longer survival than system therapy alone. ${ }^{5-10}$ Nevertheless, the role of local surgery for these patients with cervical cancer is still not established. Traditionally, since metastatic cervical cancer is considered incurable, surgical treatment is only recommended as a palliative treatment to relieve symptoms such as pain, bleeding and 
obstruction. Additionally, it has been reported that the primary tumour mass inhibits remote metastasis by a circulating angiogenesis inhibitor and once the tumour is removed, metastasis neovascularise and grow, ${ }^{11}$ but this viewpoint is not supported by clinical evidence. Growing studies have demonstrated prolonged survival in metastatic diseases when aggressive local surgeries were carried out. ${ }^{12-15}$ Venigalla et al found that patients with cervical cancer with disseminated disease would benefit from locally definitive treatment (concurrent chemotherapy or definitive surgery), and median overall survival (OS) time is elevated by 9.1 months. However, only $14 \%$ of patients in the definitive treatment group underwent definitive surgery, and the relative role of surgery on survival was not investigated. ${ }^{7}$

To further examine the benefits of primary tumour resection for patients with cervical cancer, we analysed a large amount of data from the National Cancer Institute's Surveillance, Epidemiology and End Result (SEER) programme database to conduct this population-based epidemiological study. Sites of metastasis, histological type, surgery of distant metastasis, chemotherapy, radiotherapy, T stage, lymph node (LN) status, tumour grade and epidemiological factors were stratified in the analysis of OS and cancer specific survival (CSS). We hypothesised that patients with primary metastatic cervical cancer would benefit from local surgery.

\section{METHODS}

\section{Data acquisition and processing}

We identified cervical cancer cases from the SEER database using the official software SEERStat 8.3.6 on 20 September 2019. The demographic, clinicopathological and follow-up data of 23873 women with cervical cancer diagnosed from 2010 to 2016 were obtained. After screening the data on the basis of the seventh American Joint Committee on Cancer (AJCC) staging system, 1390 patients with only one primary tumour, at least one distant metastasis, which is aligned to FIGO 2009 stage IVB, and definite information on primary site surgery were included in this study (figure 1).

Patients were classified as 'surgery' and 'no surgery' on the basis of whether they underwent primary site surgery to remove the cervical tumour after the initial diagnosis. The extent of primary site surgery was coded on the basis of the report of the operating surgeon and was categorised in the present study as 'none', 'local excision', 'simple hysterectomy', 'radical hysterectomy', and 'pelvic exenteration'. Age at diagnosis was categorised into four groups, younger than 40 years, 41-60, 61-80 and 81 and older. Based on race, patients were categorised as white, black and others. Their histological type included squamous cell carcinoma (SCC), adenocarcinoma (ADC) and others. Based on the seventh AJCC staging system, patients with $\mathrm{N} 0$ were categorised as LN negative, and N1 were positive. Distant metastases were recorded as 'bone', 'brain', 'liver', 'lung', 'distant LN', and 'other

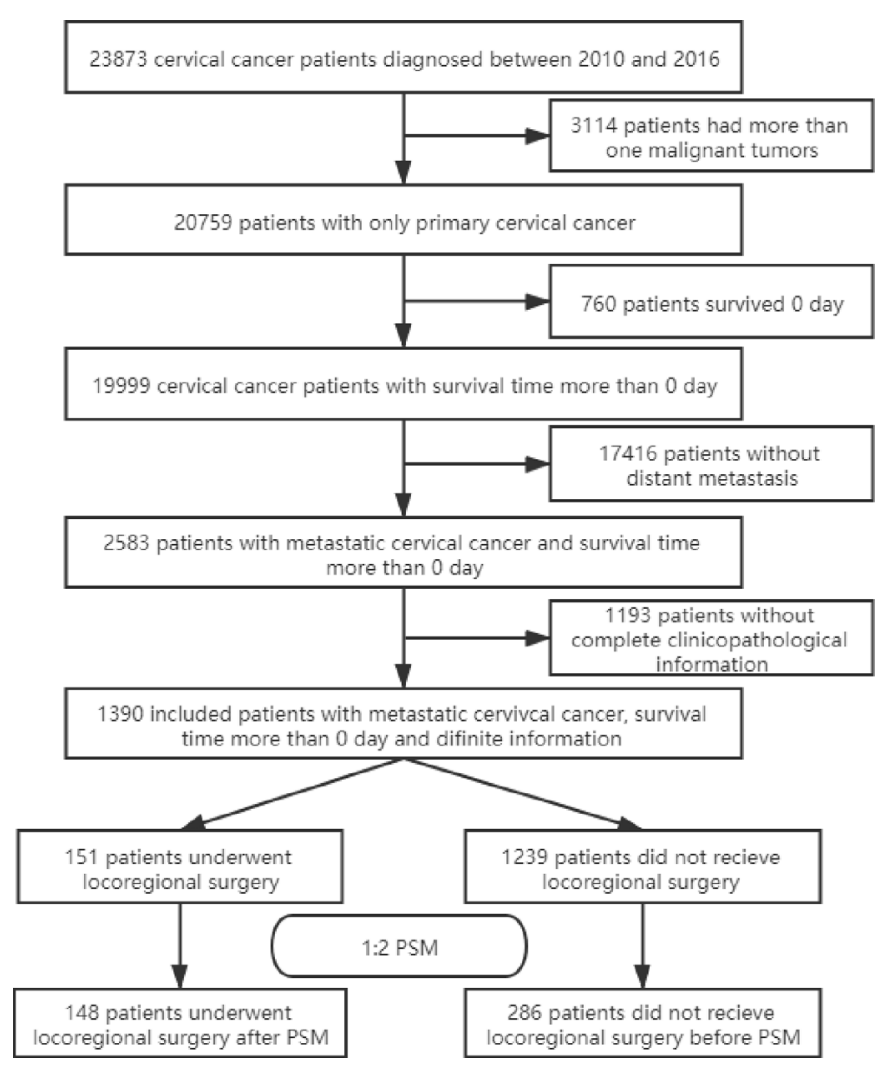

Figure 1 Flow chart. PSM, propensity score matching.

sites'. The surgery pattern of regional LN was classified as 'none' and 'lymphadenectomy'. The distant surgery was labelled as 'none' and 'yes'. Data on chemotherapy, radiation, distant surgery, insurance and marital status were extracted, but the exact regimens, cycles, dose and site were not available in the SEER database.

\section{Statistical analysis}

The primary endpoint of this observational study was to assess whether primary site surgery can improve the OS and CSS of patients with FIGO IVB cervical cancer. To adjust the potential baseline confounders, propensity score matching (PSM) was used. For the present study, all demographic and clinicopathological factors comprised the propensity matching. Propensity scores for receipt of surgery were computed with logistic regression, and matching of the propensity scores was done using a nearest-neighbour algorithm, allowing maximum tolerance between propensity scores of no larger than $20 \%$ of the propensity score standardised mean difference (SMD). A matching ratio of 1:2 was selected for surgery vs non-surgery to maximise the number of the matched pairs. Survival time was calculated using Kaplan-Meier methods and compared by the log-rank test using GraphPad Prism (V.8; GraphPad Software). For CSS, only death due to cervical cancer was considered as an event occurrence, while for OS, it was death due to any cause. Combination of directed acyclic graph (DAG) and change-in-estimate (CIE) procedures was performed to indentified confounders. ${ }^{16}$ Based 
on previous literature review and availability of information, all clinicopathological factors were potential confounders, and treatment-specific factors were intermediate variables. A DAG was created to diagram hypothesised relationships among local surgery, survival and clinicopathological factors. Variables remained in the adjusted model if variable inclusion changed the HR by greater than or equal to $10 \%$ for any given outcome. Then multivariate Cox proportional hazards models that included the local surgery and confounders were carried out to evaluate the effect of local surgery on CSS and OS using the HR for death and 95\% CI. We did not adjust for intermediate factors (treatment-specific fatcors) in order to estimate the total effect of surgery on survival. Adjusted HR for overall and cancer-specific mortality were calculated in conditional Cox regression models after PSM. Another PSM with matching ratio $1: 3$ and calliper 0.2 were performed to assess the consistency of our findings. Data analysis was performed using the software SPSS V.22.0 and Stata V.14, and a two-sided $\mathrm{p}<0.05$ was considered statistically significant.

\section{Patient and public involvement}

Patients were not study participants and were not involved in setting the research question, designing the study, in the conduct of the study or in the interpretation of the results.

\section{RESULTS}

\section{Demographic characteristics of the patients}

Among 1390 patients with FIGO IVB cervical cancer diagnosed between 2010 and 2016, 151 (10.9\%) of them underwent surgery, while $1239(89.1 \%)$ did not. The clinicopathological characteristics of patients with metastatic cervical cancer are summarised in table 1. Before PSM, the median age in the non-surgery group was 57 years, while it was 52 years in the surgery group, indicating that older patients were more prone to choose conservative treatment. Race, tumour grade and LN status did not appear to affect the decision to excise the primary site tumour. The tumours resected were less likely to be SCC and ADC, and more likely to be T1 and T2 (both SMD $>0.2$ ). The incidence of all metastasis sites did not show any differences between the two groups, except for the bone. When compared with the non-surgery group, the chances of the removal of distant metastases, and receipt of chemotherapy in the surgery group increased by $12.2 \%$ and $11.5 \%$, respectively, while the rate of receipt of radiation reduced by $11.2 \%$. The distribution of insurance and marital status showed no difference between the surgery and non-surgery groups. After PSM, the distributions of most demographical factors were significantly balanced between the two groups, except for subsequent treatment strategy after surgery (table 1). The characteristics of those patients excluded after PSM were summarised in online supplemental table 1.

\section{Analysis of CSS and OS}

To analyse the effects of local surgery on CSS and OS in patients with metastatic cervical cancer, log-rank tests were performed between the surgery and non-surgery groups. As illustrated in figure 2, local surgical treatment conferred a prominent survival advantage. The median CSS and OS were 12 and 12 months for the surgery group, and 9 and 8 months for the non-surgery group (figure 2A,B). After PSM, improvement in CSS and OS were also observed for patients receiving primary site surgery, with HR for overall and cancer-specific death were 0.70 and 0.69 (figure 2C,D).

In order to eliminate the bias of site-specific metastasis on survival analysis, data were stratified on the basis of the distant sites involved (table 2), and CSS and OS were evaluated on the basis of whether or not surgery of the primary tumour was performed in different subgroups of patients with stage IVB cervical cancer. Before matching, patients with liver, lung, distant LN and other sites involvement benefited from primary site surgery. In general, patients with one metastasis site, especially for those with isolated distant LN and other involved sites, would gain survival advantage from local surgery. Furthermore, local treatment could also prolong the survival of patients with metastasis in two or more sites. Significant survival advantages were also observed for patients with metastasis in distant LN, other sites, isolated distant LN and two or more sites after PSM. Although the $\mathrm{p}$ values were not significant for other subgroups, survival time was numerically improved. This may be caused by the insufficient sample size.

We stratified by treatment factors following surgery to eliminate the bias of intermediate variables on survival analysis (table 3). Before PSM, primary site surgery could offer overall and CSS advantage for those patients without surgery of distant metastasis sites; the advantage of primary site surgery always stay no matter whether patients received radiation or chemotherapy or not.

After matching, the survival superiority of primary site surgery did not influenced by status of distant surgery and radiation. However, primary sites surgery did not prolong the survival for patients without systemic chemotherapy, which demonstrated the vital value of chemotherapy.

Data stratified on the basis of clinicopathological factors showed that histology and LN status did not affect the survival advantage provided by local surgery. Nevertheless, we failed to observe any significant beneficial prognostic effect for patients aged $\leq 40$ years and $\geq 81$ years, those of other races, those who were uninsured and unmarried, and those who has grade I and IV, T1 and T4 tumours (figure 3).

\section{Multivariate analysis}

Multivariate analysis was then performed to evaluate the effects of local surgery on CSS and OS before and 
Table 1 Clinicopathological characteristics of women diagnosed with primary metastatic cervical cancer included in seer database before and after PSM

\begin{tabular}{|c|c|c|c|c|c|c|}
\hline & \multicolumn{3}{|l|}{ Before PSM } & \multicolumn{3}{|l|}{ After PSM } \\
\hline & Surgery (151) & No surgery (1239) & SMD & Surgery (148) & No surgery (286) & SMD \\
\hline Age (median, IQR) & $52(44-65)$ & $57(47-66)$ & 0.203 & $53(44-65)$ & $54(43-62)$ & 0.040 \\
\hline Race $(n, \%)$ & & & 0.082 & & & 0.025 \\
\hline White & 109 (72.2) & 891 (71.9) & & $106(71.6)$ & 208 (72.7) & \\
\hline Black & 25 (16.6) & $233(18.8)$ & & 25 (16.9) & $46(16.1)$ & \\
\hline Others & $17(11.3)$ & $115(9.3)$ & & $17(11.5)$ & $32(11.2)$ & \\
\hline Histology (n, \%) & & & 0.457 & & & 0.047 \\
\hline SCC & $66(43.7)$ & $803(64.8)$ & & 66 (44.6) & $128(44.8)$ & \\
\hline ADC & $41(27.2)$ & $257(20.7)$ & & $41(27.7)$ & 74 (25.9) & \\
\hline Others & $44(29.1)$ & $179(14.4)$ & & $41(27.7)$ & $84(29.9)$ & \\
\hline Grade (n, \%) & & & 0.166 & & & 0.100 \\
\hline 1 & $12(7.9)$ & $73(5.9)$ & & $12(8.1)$ & $28(9.8)$ & \\
\hline II & 48 (31.8) & 331 (26.7) & & $46(31.1)$ & 78 (27.3) & \\
\hline III & $72(47.7)$ & $688(55.5)$ & & $71(48.0)$ & $145(50.7)$ & \\
\hline IV & $19(12.6)$ & $147(11.9)$ & & $19(12.8)$ & 35 (12.2) & \\
\hline T-stage (n, \%) & & & 0.330 & & & 0.126 \\
\hline $\mathrm{T} 1$ & $32(21.2)$ & $180(14.5)$ & & $31(20.9)$ & $58(20.3)$ & \\
\hline T2 & $41(27.2)$ & $274(22.1)$ & & $40(27.0)$ & $82(28.7)$ & \\
\hline T3 & $46(30.5)$ & $570(46.0)$ & & $46(31.1)$ & 99 (34.6) & \\
\hline T4 & $32(21.2)$ & $215(17.4)$ & & $31(20.9)$ & $47(16.4)$ & \\
\hline N stage (n, \%) & & & 0.058 & & & 0.011 \\
\hline NO & $53(35.1)$ & $401(32.4)$ & & $51(34.5)$ & $102(35.0)$ & \\
\hline $\mathrm{N} 1$ & $98(64.9)$ & $838(67.6)$ & & $97(65.5)$ & $186(65.0)$ & \\
\hline \multicolumn{7}{|c|}{ Distant metastasis (n, \%) } \\
\hline Bone & $33(21.9)$ & $415(33.5)$ & 0.262 & 33 (22.3) & $62(21.7)$ & 0.015 \\
\hline Brain & $5(3.3)$ & $63(5.1)$ & 0.089 & $5(3.4)$ & $12(4.2)$ & 0.043 \\
\hline Lung & $71(47.0)$ & $706(57.0)$ & 0.200 & $70(47.3)$ & $156(54.5)$ & 0.145 \\
\hline Liver & $39(25.8)$ & 354 (28.6) & 0.062 & $38(25.7)$ & $65(22.7)$ & 0.069 \\
\hline Distant LN & $91(60.3)$ & $749(60.5)$ & 0.004 & $91(61.5)$ & $175(61.2)$ & 0.006 \\
\hline Other sites & $51(33.8)$ & $343(27.7)$ & 0.132 & $48(32.4)$ & 91 (31.8) & 0.013 \\
\hline Distant surgery (n, \%) & & & 0.383 & & & 0.412 \\
\hline No & $124(82.1)$ & 1168 (94.3) & & $121(81.8)$ & $271(94.8)$ & \\
\hline Yes & 27 (17.9) & $71(5.7)$ & & 27 (18.2) & $15(5.2)$ & \\
\hline Chemotherapy (n, \%) & & & 0.276 & & & 0.218 \\
\hline No/unknown & $26(17.2)$ & $356(28.7)$ & & $26(17.6)$ & $76(26.6)$ & \\
\hline Yes & $125(82.8)$ & $883(71.3)$ & & $122(82.4)$ & $210(73.4)$ & \\
\hline Radiation (n, \%) & & & 0.228 & & & 0.161 \\
\hline No & $76(50.3)$ & $484(39.1)$ & & $75(50.7)$ & $120(42.7)$ & \\
\hline Yes & $75(49.7)$ & 755 (60.9) & & $73(49.3)$ & $164(57.3)$ & \\
\hline Insurance (n, \%) & & & 0.031 & & & 0.019 \\
\hline No & $10(5.6)$ & $92(7.4)$ & & $10(6.8)$ & $18(6.3)$ & \\
\hline Yes & 141 (93.4) & 1147 (92.6) & & 138 (93.2) & 268 (93.7) & \\
\hline Marital status (n, \%) & & & 0.051 & & & 0.068 \\
\hline No & 50 (33.1) & $440(35.5)$ & & 49 (33.1) & $104(36.4)$ & \\
\hline
\end{tabular}


Table 1 Continued

\begin{tabular}{llllllll}
\hline & \multicolumn{3}{l}{ Before PSM } & & & \multicolumn{2}{l}{ After PSM } \\
\cline { 2 - 3 } & Surgery (151) & No surgery (1239) & SMD & & Surgery (148) & No surgery (286) & SMD \\
\hline Yes & $101(66.9)$ & $799(64.5)$ & $99(66.9)$ & $182(63.6)$ & \\
\hline
\end{tabular}

ADC, adenocarcinoma; LN, lymph node; PSM, propensity score matching; SCC, squamous cell carcinoma; SMD, standardised mean difference.

after PSM. The combination of DAG and CIE was used to identify the confounders and intermediate variables. An explanatory theoretical model was built based on clinicopathological variables established in the literature that could have some influence on the association between local surgery and survival, which was subsequently analysed using DAG. The interrelations between these variables are represented in online supplemental figure 2. All clinicopathological variables were assumed to be related to the exposure variable and the outcome variable, being potential confounders and are represented in green colour. While treatment-specific factors were assumed as intermediate variables and are presented in black colour. Then Cox proportional hazards models were created to assess change in HR estimate for CSS or OS corresponding to all potential confounders (table 4). The order of confounders were entered into the model was based on their expected association with survival. For CSS and OS before PSM, the HR for death from the crude model changed less than $10 \%$ after inclusion of all potential confounders. Similarly, after PSM, inclusion of all potential confounders did not change HR for death of CSS and OS more than $10 \%$. Therefore, no factor was confounder for OS and CSS before and after PSM. Before PSM, local surgery could reduced the cancer-associated and overall mortality rates by $40 \%$ (both HR $0.60,95 \%$ CI, 0.48 to $0.76, \mathrm{p}<0.0001)$. After PSM, local surgery could reduced the cancer-associated and overall mortality rates by $31 \%$ (HR $0.69,95 \%$ CI, 0.53 to $0.90, \mathrm{p}=0.007$ ) and $30 \%$ (HR $0.70,95 \%$ CI 0.54 to $0.91, \mathrm{p}=0.008$ ), respectively. It was concluded that local resection of the primary tumour is an independent prognosis factor and is of vital significance in prolonging the survival of patients with cervical cancer with primary metastasis. As for the local surgical pattern, radical hysterectomy conferred better CSS and OS when compared with non-surgery before and after matching (before PSM, HR for death were $0.46,95 \%$ CI, 0.31 to 0.68 , and $0.48,95 \%$ CI, 0.33 to 0.70 ; after PSM, HR for death were $0.53,95 \% \mathrm{CI}, 0.35$ to 0.80 and 0.56 , $95 \%$ CI, 0.38 to 0.83$)$. However, local excisions, simple
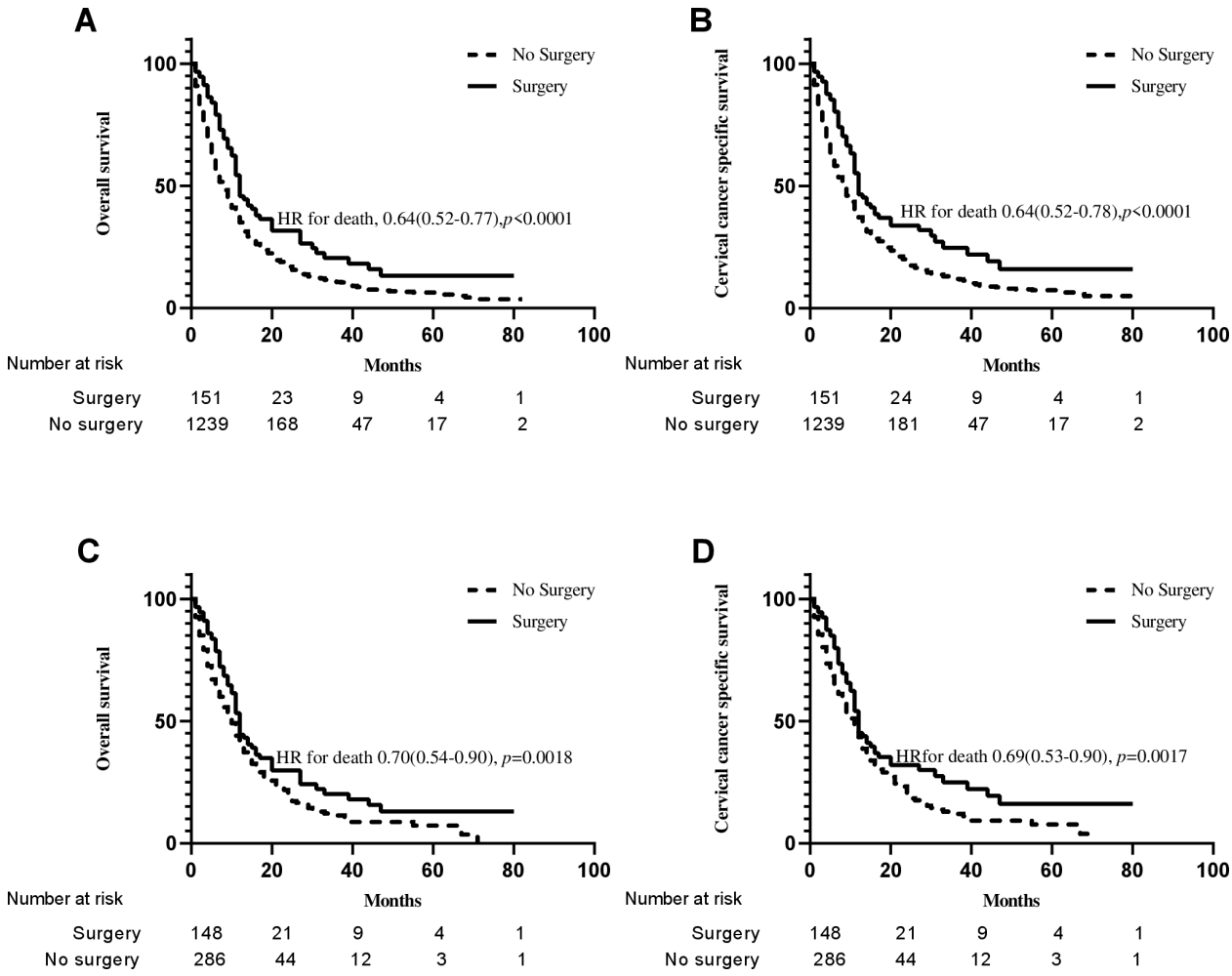

Figure 2 Cancer-specific survival (CSS) and overall survival (OS) of patients with FIGO IVB cervical cancer before and after PSM. (A, B) CSS and OS in the surgery and no-surgery groups before PSM. (C, D) CSS and OS in the surgery and no-surgery groups after PSM. FIGO, Federation of Gynaecology and Obstetrics; PSM, propensity score matching. 


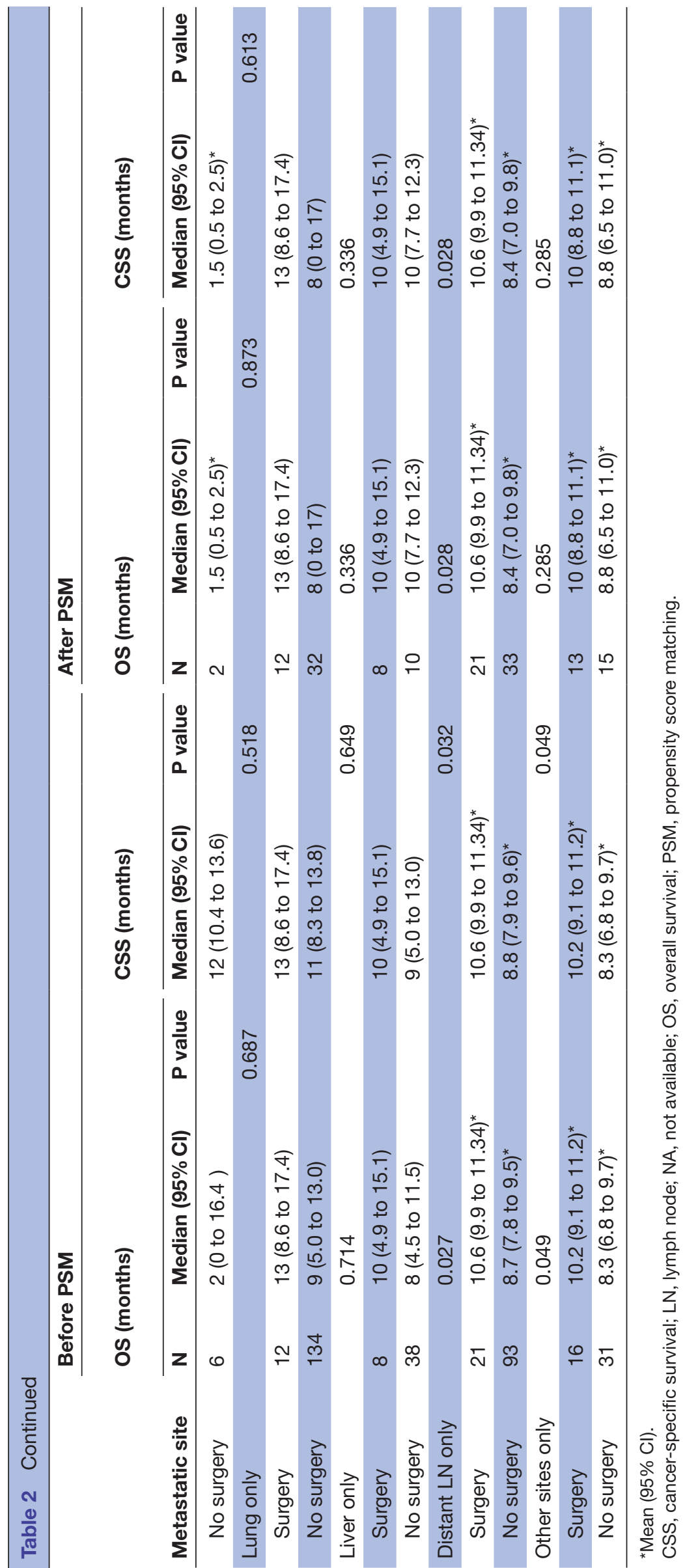




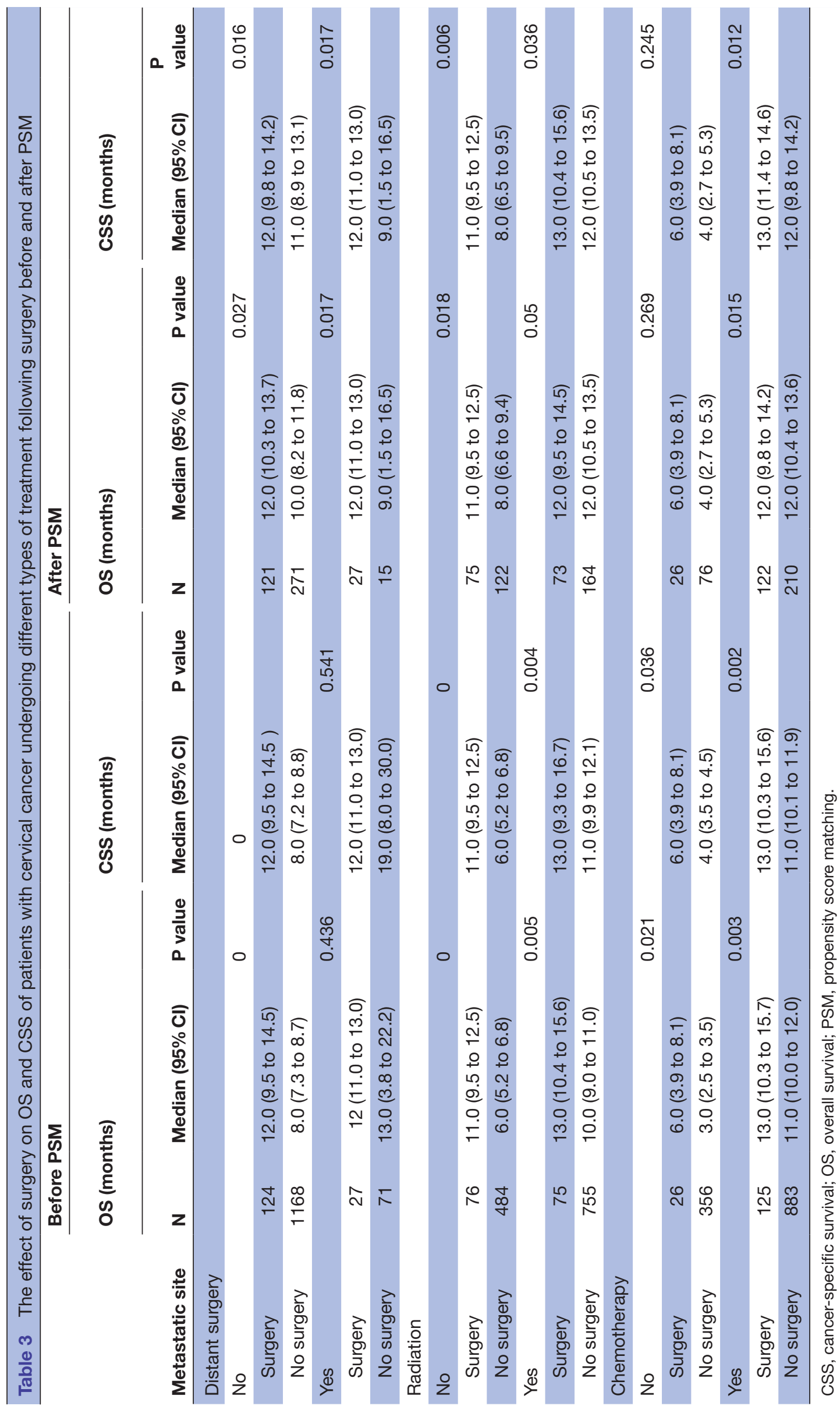




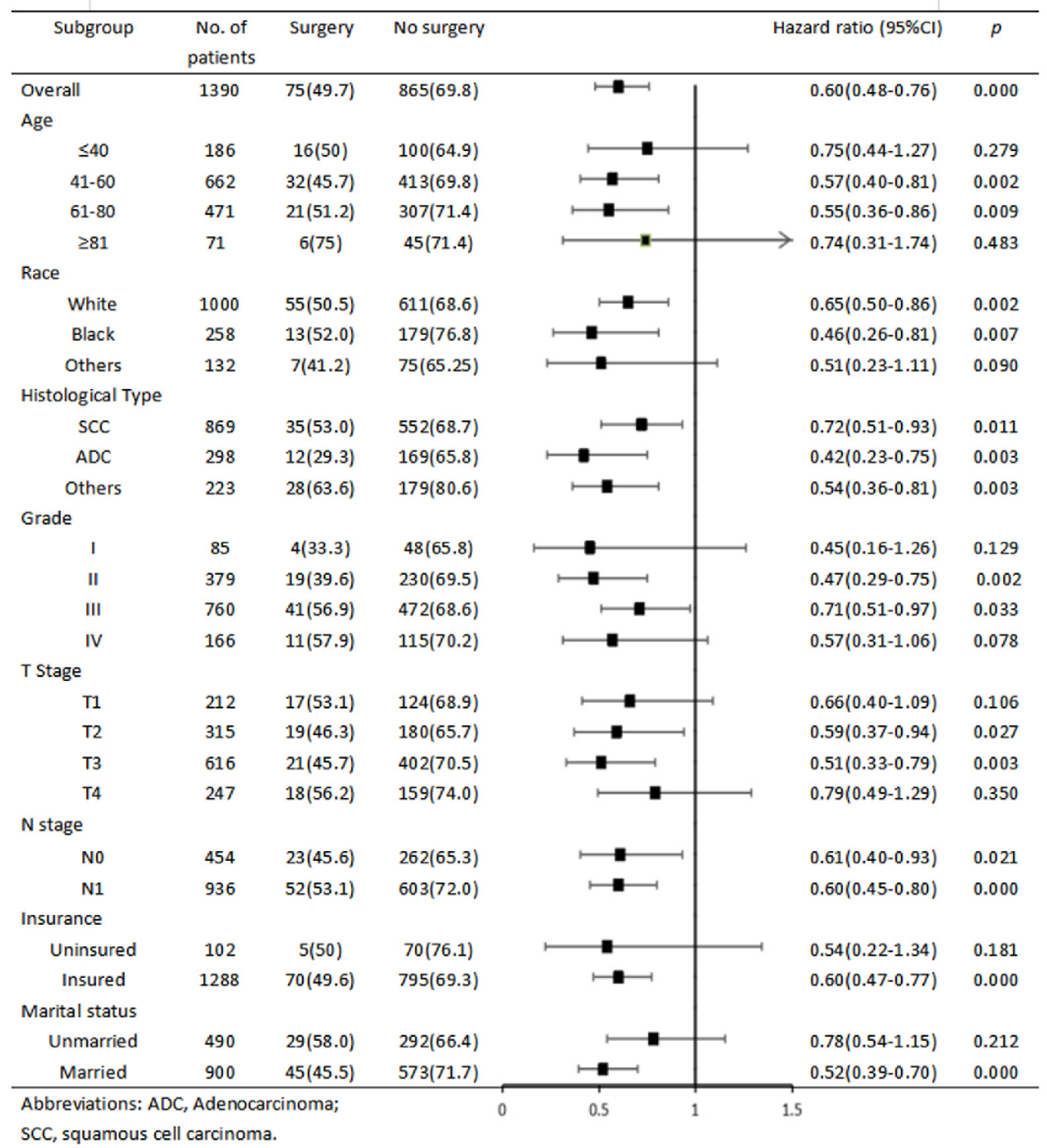

Figure 3 Forest plot summarising HR for cervical cancer-specific death and $95 \%$ Cls in subgroup received primary site surgery or not before PSM. PSM, propensity score matching.

hysterectomy and pelvic exenterations showed no effect on survival.

\section{Sensitivity analysis}

Another PSM with matching ratio 1:3 was conducted, and the distributions of most demographical factors were well balanced between the two groups (online supplemental table 2). Similar findings were observed. Statistically significant reduction in overall and cancer specific mortality with local surgery were observed (OS: HR, 0.70, 95\% CI, 0.55 to $0.89, \mathrm{p}=0.0008$; CSS: HR, 0.70, 95\% CI, 0.55 to $0.89, \mathrm{p}=0.0008$, online supplemental figure $1 \mathrm{~A}, \mathrm{~B})$.

\section{DISCUSSION}

Usually, the treatment for FIGO IVB cervical cancer has been and continues to be considered palliative. Moreover, systemic therapies including platinum-based chemotherapy, targeted therapy and immunotherapy have been mainstay of care for patients with metastatic cervical cancer. ${ }^{3}$ In recent years, the efficacy of locoregional management on improving survival in M1 cervical cancer has been highlighted in multiple retrospective studies. A retrospective study by Sasano $\mathrm{T}$ et al indicated that treatment with definitive radiotherapy was associated with improved survival compared with chemotherapy or palliative care alone. ${ }^{2}$ Another study by Sriram et $a l$, with a large sample size of 2838 identified from the National Cancer Database, revealed that patients who underwent definitive local therapy (either concurrent chemoradiation or surgery) had a $43 \%$ reduced mortality risk compared with those who received conservative therapy (systemic therapy with or without palliative radiation). ${ }^{7}$ A similar conclusion was drawn by a Chinese study, which illustrated that chemotherapy combined with definitive pelvic radiotherapy would significantly prolong the OS by 7.3 months when compared with chemotherapy alone or with palliative pelvic radiotherapy. ${ }^{5}$ Another two studies, which enrolled patients from SEER or the National Cancer Database, also demonstrated that radiotherapy would decrease $28 \%-31 \%$ mortality risk for primary metastatic cervical 
Table 4 Change in HR estimates for CSS and OS for stepwise inclusion of clinicopathological factors into Cox proportional hazards models before and after PSM

\begin{tabular}{|c|c|c|c|c|c|c|c|c|}
\hline & \multicolumn{4}{|l|}{ Before PSM } & \multicolumn{4}{|l|}{ After PSM } \\
\hline & \multicolumn{2}{|l|}{ css } & \multicolumn{2}{|l|}{ os } & \multicolumn{2}{|l|}{ css } & \multicolumn{2}{|l|}{ os } \\
\hline & HR (95\% CI) & $\begin{array}{l}\Delta \\
\mathrm{HR}(\%)\end{array}$ & HR $(95 \% \mathrm{Cl})$ & $\begin{array}{l}\Delta \\
\mathrm{HR}(\%)\end{array}$ & HR $(95 \% \mathrm{Cl})^{*}$ & $\begin{array}{l}\Delta \\
\mathrm{HR}(\%)\end{array}$ & HR $(95 \% \mathrm{Cl})^{*}$ & $\begin{array}{l}\Delta \\
\mathrm{HR}(\%)\end{array}$ \\
\hline Local surgery & 0.60 (0.48 to 0.76$)$ & & $0.60(0.48$ to 0.76$)$ & & 0.69 (0.53 to 0.90$)$ & & 0.70 (0.54 to 0.91$)$ & \\
\hline +Liver & 0.60 (0.48 to 0.76$)$ & 0 & 0.61 (0.48 to 0.76$)$ & 1.7 & 0.69 (0.54 to 0.90$)$ & 0 & 0.70 (0.54 to 0.91$)$ & 0 \\
\hline +Lung & $0.61(0.49$ to 0.78$)$ & 1.7 & 0.62 (0.49 to 0.77$)$ & 1.6 & 0.70 (0.54 to 0.90$)$ & 1.4 & 0.71 (0.54 to 0.92$)$ & 1.4 \\
\hline +Bone & 0.64 (0.50 to 0.81$)$ & 3.2 & 0.64 (0.51 to 0.80$)$ & 3.2 & 0.70 (0.53 to 0.92$)$ & 1.4 & 0.71 (0.55 to 0.93$)$ & 1.4 \\
\hline +Other sites & 0.64 (0.51 to 0.81$)$ & 0 & 0.64 (0.51 to 0.80$)$ & 0 & 0.70 (0.54 to 0.92$)$ & 0 & 0.70 (0.54 to 0.92$)$ & 1.4 \\
\hline +Distant LN & 0.64 (0.51 to 0.81$)$ & & 0.64 (0.51 to 0.80$)$ & 0 & 0.70 (0.53 to 0.92$)$ & 0 & 0.70 (0.54 to 0.91$)$ & 0 \\
\hline +T stage & 0.65 (0.51 to 0.83$)$ & 1.6 & 0.65 (0.52 to 0.82$)$ & 1.6 & 0.70 (0.54 to 0.92$)$ & 0 & 0.70 (0.54 to 0.92$)$ & 0 \\
\hline+ Age & 0.67 (0.52 to 0.85$)$ & 3.1 & 0.67 (0.53 to 0.89$)$ & 3.1 & 0.67 (0.51 to 0.88$)$ & 2.9 & 0.67 (0.51 to 0.87$)$ & 2.9 \\
\hline +Race & 0.67 (0.52 to 0.85$)$ & 0 & 0.67 (0.53 to 0.85$)$ & 0 & 0.67 (0.51 to 0.88$)$ & 0 & 0.67 (0.51 to 0.87$)$ & 0 \\
\hline +Insurance & 0.67 (0.53 to 0.86$)$ & 0 & 0.68 (0.54 to 0.86$)$ & 1.5 & 0.67 (0.51 to 0.88$)$ & 0 & 0.67 (0.51 to 0.87$)$ & 0 \\
\hline +Marital status & 0.67 (0.53 to 0.86$)$ & 0 & 0.68 (0.54 to 0.86$)$ & 0 & $0.67(0.51$ to 0.88$)$ & 0 & 0.67 (0.51 to 0.87$)$ & 0 \\
\hline
\end{tabular}

$\Delta \mathrm{HR} \%=|\mathrm{HR} 1-\mathrm{HR} 2| / \mathrm{HR} 1$.

${ }^{*}$ Adjusted HR based on conditional Cox regression model.

CSS, cancer-specific survival; LN, lymph node; OS, overall survival; PSM, propensity score matching.

cancer. ${ }^{68}$ However, in the literature, primary tumour resection in patients with FIGO IVB cervical cancer is poorly documented. In this study, based on the SEER database, we found that excision of the primary cervical tumour, in general, could bring a survival benefit for metastatic cervical cancer, reducing the risk of mortality by about $30 \%$ and prolonging the median survival by $3-4$ months, which is similar to the findings in a previous report of radiotherapy. ${ }^{68}$ Several plausible reasons might help to explain why local surgery would contribute to improving the prognosis in patients with metastatic breast cancer. First, local surgery could mitigate symptoms such as pain, bleeding and infection, intrapelvic disease burden, and gastrointestinal/genitourinary compression, which pose a risk of morbidity and mortality that is independent of distant disease. ${ }^{17}$ However, systemic therapy alone is often insufficient to address such consequences of local progression. Second, primary site control may reduce the risk of further seeding of malignant cells and thereby reduce the risk of developing new distant dissemination. ${ }^{7}$ Third, surgical removal of the primary tumour decreases the production of immunosuppressive cytokines, thereby reversing tumour-induced immunosuppression and allowing the host immune system to regain immunological control of systemic disease burden. ${ }^{18}$ Fourth, surgery removes the necrotic centric areas that are hypoxic and resistant to chemotherapy and radiotherapy and, therefore, increases the efficacy of adjuvant treatment and improves prognosis. ${ }^{19-21}$
Although local surgery poses a benefit of survival, it is worth mentioning that such therapy is likely not suitable for all patients with metastatic disease. The stratified analysis after PSM in this study demonstrated that hysterectomy resulted in survival improvement in patients with cervical cancer with metastasis to distant LN and other sites. In line with previous studies, patients with metastasis to distant LN (such as paraortic or supraclavicular LN) have remarkably good prognosis and prolonged survival from aggressive therapy. ${ }^{10} 2223$ Nevertheless, metastatic tumour burden should be taken into account. We found that local surgery could still bring marginal improvements in CSS and OS for patients with two or more metastasis sites, which would be defined as polymetastatic disease ( $p=0.060$ and 0.048 ). It is well known that a clear surgical margin is important for survival. ${ }^{24}{ }^{25}$ Unfortunately, data on surgical margin status were not available in the SEER database. However, we found that there was no advantage of surgery in patients with grade IV tumour and T4 stage (invasion into neighbouring structures such as bladder and rectum). Moreover, patients could benefit from radical hysterectomy but not local excision and simple hysterectomy. The potential reason may lie in that a clear surgical margin is difficult to achieve for patients with tumours progression to neighbouring organs and who are receiving local excision or simple hysterectomy. An undifferentiated tumour is more prone to invasion to neighbouring organs and 
metastasis dissemination, which makes it more difficult to remove the tumour precisely. Given the radiosensitive nature of cervical cancer and the positive role for metastasis cervical cancer, ${ }^{5-10}$ radiation can be proposed to patients who do not have an emergency (bleeding, pain, and obstruction) and have an extensive locoregional tumour. Local surgery to remove a primary cervical tumour with a clear surgical margin may be an appropriate treatment option for a carefully selected group of patients with a good performance status, symptomatic primary disease, and limited local disease burden, especially for those with metastasis to distant LN, or other sites, or two and more sites.

There are several inevitable limitations to the present study. First, there is an inherent patient selection bias as this is a population-based retrospective study. We used PSM to reduce the bias caused by the imbalance of measured covariates. Nevertheless, bias from unmeasured factors is unavoidable. Sensitivity analysis was also performed to assess the validity of our findings. Second, the details regarding how metastasis was diagnosed were unavailable and may affect the result to some extent. The participants in our study only had one primary tumour, namely cervical cancer, with an active follow-up survival time of more than 1 month, which could help differentiate patients with metastases from other cancers. Third, it is preferable to get more details regarding their surgery in correlation to potential survival benefit, such as the surgical margin and recovery time following surgery. However, such information was not available in SEER database. A prospective study is warranted to ascertain the value of recovery time. Fourth, aspects of the systemic therapy and radiotherapy, such as the type of radiotherapy (RT), dose, intent, regimen and cycles of chemotherapy were lacking, which could also affect prognosis. In the current analysis, we did not intend to demonstrate the effect of RT or chemotherapy on survival. Fifth, the SEER database contains metastatic information for patients with cervical cancer patients only after 2010; therefore, our sample size was small. A prospective or retrospective study with larger sample size was need in future.

\section{CONCLUSION}

Surgical procedure exerts some positive effects on patients having merely metastasis to one site, especially for isolated distant LN, and having a small and nonundifferentiated primary tumour. Further well-designed studies should be conducted to identify the advantage of surgery over palliative therapy; furthermore, the surgical margin and recovery time following surgery, systemic treatment strategy and diagnosis method of metastasis deserve more attention.

Acknowledgements All authors thank Editage for its linguistic assistance during the preparation of this manuscript.
Contributors YW: project development and manuscript writing. YO: data collection and manuscript writing. JS: data collection. ZB: data analysis. QC: project development and manuscript review and editing. XC: project development and manuscript review and editing.

Funding This work was supported by Science and Technology Planning Project of Quanzhou Science and Technology Bureau grant number (2018N001S) and Fujian Provincial Health and Family planning Commission Research Talent training project grant number (2018-1-60).

Competing interests None declared.

Patient consent for publication Not required.

Ethics approval The SEER database was publicly available, and all the research data were de-identified. Therefore, ethical approval and informed consent were not required in this study.

Provenance and peer review Not commissioned; externally peer reviewed.

Data availability statement Data are available on reasonable request. The data that support the findings of this study are available on request from the corresponding author. The data that support the findings of this study are available on reasonable request from the corresponding author.

Supplemental material This content has been supplied by the author(s). It has not been vetted by BMJ Publishing Group Limited (BMJ) and may not have been peer-reviewed. Any opinions or recommendations discussed are solely those of the author(s) and are not endorsed by BMJ. BMJ disclaims all liability and responsibility arising from any reliance placed on the content. Where the content includes any translated material, BMJ does not warrant the accuracy and reliability of the translations (including but not limited to local regulations, clinical guidelines, terminology, drug names and drug dosages), and is not responsible for any error and/or omissions arising from translation and adaptation or otherwise.

Open access This is an open access article distributed in accordance with the Creative Commons Attribution Non Commercial (CC BY-NC 4.0) license, which permits others to distribute, remix, adapt, build upon this work non-commercially, and license their derivative works on different terms, provided the original work is properly cited, appropriate credit is given, any changes made indicated, and the use is non-commercial. See: http://creativecommons.org/licenses/by-nc/4.0/.

ORCID iD

Yanhong Wang http://orcid.org/0000-0001-8152-0574

\section{REFERENCES}

1 Siegel RL, Miller KD, Jemal A. Cancer statistics, 2017. CA Cancer J Clin 2017;67:7-30.

2 Sasano T, Mabuchi S, Kuroda H, et al. Predictors of survival in patients with FIGO stage IVb cervical cancer. Int J Gynecol Cancer 2016;26:528-33.

3 Cohen PA, Jhingran A, Oaknin A, et al. Cervical cancer. Lancet 2019;393:169-82.

4 Waggoner SE. Cervical cancer. Lancet 2003;361:2217-25.

5 Yin Z, Lou H, Tang H, et al. Efficacy of radical doses of pelvic radiotherapy for primary tumor treatment in patients with newly diagnosed organ metastatic cervical cancer. Radiat Oncol 2019;14:82.

6 Wang Y, Farmer M, Izaguirre EW, et al. Association of definitive pelvic radiation therapy with survival among patients with newly diagnosed metastatic cervical cancer. JAMA Oncol 2018;4:1288-91.

7 Venigalla S, Guttmann DM, Horne ZD, et al. Definitive local therapy is associated with improved overall survival in metastatic cervical cancer. Pract Radiat Oncol 2018;8:e377-85.

8 Huang K, Jia M, Li P, et al. Radiotherapy improves the survival of patients with metastatic cervical cancer: a Propensity-Matched analysis of seer database. Int J Gynecol Cancer 2018;28:1360-8.

9 Hata M, Koike I, Miyagi E, et al. Radiation therapy for patients with bone metastasis from uterine cervical cancer: its role and optimal radiation regimen for palliative care. Anticancer Res 2018;38:1033-40.

$10 \mathrm{Kim} \mathrm{J}-\mathrm{Y}, \mathrm{Kim} \mathrm{J}-\mathrm{Y}, \mathrm{Kim} \mathrm{JH}$, et al. Curative chemoradiotherapy in patients with stage IVb cervical cancer presenting with paraortic and left supraclavicular lymph node metastases. Int J Radiat Oncol Biol Phys 2012;84:741-7.

11 O'Reilly MS, Holmgren L, Shing Y, et al. Angiostatin: a novel angiogenesis inhibitor that mediates the suppression of metastases by a Lewis lung carcinoma. Cell 1994;79:315-28. 
12 Shi X, Huang N-S, Shi R-L, et al. Prognostic value of primary tumor surgery in minor salivary-gland carcinoma patients with distant metastases at diagnosis: first evidence from a SEER-based study. Cancer Manag Res 2018:10:2163-72.

13 Luo D, Liu Q, Yu W, et al. Prognostic value of distant metastasis sites and surgery in stage IV colorectal cancer: a population-based study. Int J Colorectal Dis 2018;33:1241-9.

14 Chen J, Kong Y, Weng S, et al. Outcomes of surgery for gastric cancer with distant metastases: a retrospective study from the seer database. Oncotarget 2017;8:4342-51.

15 Rafii A, Deval B, Geay J-F, et al. Treatment of FIGO stage IV ovarian carcinoma: results of primary surgery or interval surgery after neoadjuvant chemotherapy: a retrospective study. Int J Gynecol Cancer 2007; 17:777-83.

16 Evans D, Chaix B, Lobbedez T, et al. Combining directed acyclic graphs and the change-in-estimate procedure as a novel approach to adjustment-variable selection in epidemiology. BMC Med Res Methodol 2012;12:156.

17 Ramondetta L. What is the appropriate approach to treating women with incurable cervical cancer? J Natl Compr Canc Netw 2013;11:348-55.

18 Danna EA, Sinha P, Gilbert M, et al. Surgical removal of primary tumor reverses tumor-induced immunosuppression despite the presence of metastatic disease. Cancer Res 2004;64:2205-11.
19 Lara PC, Lloret M, Clavo B, et al. Severe hypoxia induces chemoresistance in clinical cervical tumors through MVP over-expression. Radiat Oncol 2009;4:29.

20 Markowska J, Grabowski JP, Tomaszewska K, et al. Significance of hypoxia in uterine cervical cancer. multicentre study. Eur J Gynaecol Oncol 2007;28:386-8.

21 Rofstad EK, Sundfør K, Lyng H, et al. Hypoxia-Induced treatment failure in advanced squamous cell carcinoma of the uterine cervix is primarily due to hypoxia-induced radiation resistance rather than hypoxia-induced metastasis. Br J Cancer 2000;83:354-9.

22 loffe YJ, Massad LS, Powell MA, et al. Survival of cervical cancer patients presenting with occult supraclavicular metastases detected by FDG-Positron emission Tomography/CT: impact of disease extent and treatment. Gynecol Obstet Invest 2018;83:83-9.

23 Dabi Y, Simon V, Carcopino X, et al. Therapeutic value of surgical paraaortic staging in locally advanced cervical cancer: a multicenter cohort analysis from the FRANCOGYN Study Group. J Transl Med 2018;16:326.

24 McCann GA, Taege SK, Boutsicaris CE, et al. The impact of close surgical margins after radical hysterectomy for early-stage cervical cancer. Gynecol Oncol 2013;128:44-8.

25 Khanna N, Rauh LA, Lachiewicz MP, et al. Margins for cervical and vulvar cancer. J Surg Oncol 2016;113:304-9. 